\title{
Clinical Isokinetic Study Comparing Two Techniques for Reconstructing the Anterior Cruciate Ligament Using an Autologous Transplant Taken from the ST4 Versus STG
}

\author{
Joly B*, Grosset A, Chapon MP, Borrini L, Lapeyre E, Rigal S and Mathieu L \\ Service de chirurgie orthopédique, Traumatologie et chirurgie réparatrice, Europe \\ *Corresponding author: Joy B, Service de chirurgie orthopédique, traumatologie et chirurgie réparatrice, Europe \\ Submission: 眥 January 29, 2018; Published: 嗃 February 06, 2019
}

\begin{abstract}
Purpose: Two techniques of anterior cruciate ligament (ACL) reconstruction using hamstring tendons are compared: a technique using only the semitendinosus tendon (ST4) with a specific fixation using the TLS system (Tape Locking Screw(-FH Orthopaedic(C) and a technique using the semitendinosus and gracilis tendons (STG) with conventional fixation (femoral endobutton and tibial interference screw).
\end{abstract}

Materials and method: A retrospective study was conducted in the orthopaedic department of a french military hospital between January 2014 and March 2016. All of the patients who were treated with ACL ligamentoplasty to the pes anserinus tendons were included. They were divided into two groups according to the technique used: ST4 or STG. The results were evaluated through an isokinetic muscle test at 6 months, then using the IKDC score at 6,12 , and 18 months.

Results: Sixty-nine patients were included: 36 in the ST4 group and 33 in the STG group. The hamstring deficit (H) was $19.5 \%$ in the ST4 group, and $21.3 \%$ in the STG group. The quadriceps deficit (Q) was 9\% in the ST4 group, and 10.8\% in the STG group. The IKDC score at 12 months was 87.2 in the ST 4 group, and 85.5 in the STG group. No significant differences were shown between the two groups.

Conclusion: The functional results appear to be slightly better in the ST4 group, though not to a significant level. The isokinetic H/Q ratio and the conditions of resuming sports practice were satisfying and comparable in both groups. ACL ligamentoplasty using only the semitendinosus tendon seems to be reliable, even for military patients who are subject to specific operational constraints.

Keywords: Isokinetics; Semitendinosus; Anterior cruciate ligament; Military

\section{Introduction}

Anterior cruciate ligament (ACL) tears are a commonly-seen injury in sports traumatology, particularly among soldiers, who have specific operational constraints. Autograft reconstruction techniques using the hamstring tendons are usually based on grafts harvested from two tendons, the semitendinosus and the gracilis (STG). They provide good post-operative stability but carry the risk of a loss of strength when the knee is flexed [1,2]. To reduce this side-effect, some suggest only harvesting a single tendon: the semitendinosus tendon [3].

The main purpose of this study was to compare the results obtained using the classic, two-tendon technique versus a technique using a single tendon, particularly by evaluating their impact on the muscle strength of the hamstrings and the quadriceps $[4,5]$. The hypothesis was that the technique using only the semitendinosus would produce clinical and isokinetic results comparable to the STG technique. The secondary purpose was to assess the conditions of resuming sports and professional activities one year after surgery, particularly in military patients [6].

The goal of our study was to search for differences in muscle strength in the hamstrings and quadriceps between the two hamstring reconstruction techniques (ST4-TLS versus STG). Isometric testing was performed at 6 months post-operation using a dynamometer $[4,5]$. We also wanted to look for a correlation between the IKDC score and muscular deficits.

\section{Materials and Method}

A retrospective, monocentric, multi-surgeon study was carried out between January 2014 and March 2016 in the orthopaedic department of a French military hospital. During this period, all patients who underwent primary, isolated ACL ligamentoplasty using an autograft transplant harvested from the hamstring were included. The study excluded patients that received a patellar tendon ligamentoplasty, patients that required associated ligamentous reconstruction (particularly of the posterior cruciate ligament or 
the lateral plane), patients presenting an ACL tear that had already been reconstructed (transplant tear), and patients having an injury to the contralateral ACL (regardless of operative status).

\section{Operating procedure}

In both groups the ACL was reconstructed under arthroscopy with or without treatment of the associated meniscus tears and chondral injuries. In the ST4 group, the semitendinosus was braided to create a short, 4-stranded transplant that was implanted according to the "all in" technique, then fixed in place with the TLS system (Tape Locking Screw (C) from the company FH Orthopedics (C) (Figure 1). In the STG group, the semitendinosus and the gracilis were braided into a long, 4-stranded transplant that was also implanted according to the "all in" technique. The STG was fixed in place with a femoral endobutton (Endobutton-CL@), Smith $\&$ Nephew (C) and a resorbable interference screw (Biosure-PK(C), Smith \& Nephew(C) in the tibia. The post-operative rehabilitation protocol was identical in both groups and consisted of three weeks hospitalisation in a functional rehabilitation department. Effort training and the resumption of sports were preceded by isokinetic muscle testing.

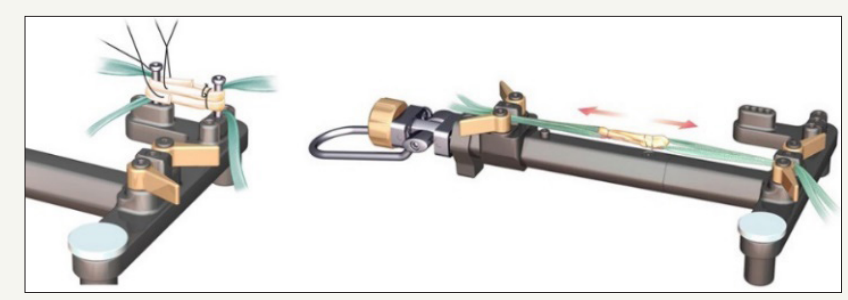

Figure 1: Preparation and fixation of the ST4-TLSC system composed of polyethylene terephthalate strips and two titanium screws.

\section{Parameters studied}

The demographic variables gathered were the sex, age, body mass index (BMI), military status, and levels of sports practice. The perioperative data studied were the time between the injury and the ligamentoplasty, the pre-operative IKDC score (International Knee Documentation Committee), the presence of associated meniscus tears and chondral injuries and their treatment, the diameter of the transplant, and the occurrence of post-operative complications.

Isokinetic assessment was performed at 6 months post-op using a CYBEX 2 dynamometer to create groups of ability levels during an effort training course according to the degree of muscular deficit. The protocol consisted of an isokinetic test of the extensors and flexors of both knee, concentrically and eccentrically, at three different speeds: slow $\left(30^{\circ} / \mathrm{sec}\right)$, medium $\left(60^{\circ} / \mathrm{sec}\right)$, and fast $\left(240^{\circ} \%\right.$ sec). The three series were spaced with one minute of rest. The test was interpretable in the absence of pain, and if the coefficient of variation of the peak torque was less than 10\% [7]. According to Croisier et al. [8], isokinetic assessment should bring out a dynamic equilibrium between the antagonist muscles by calculating the eccentric $H$ deficit ratio at a slow speed over the concentric $Q$ deficit at a high speed. This ratio must be between 0.8 and 1 [9] in order to obtain a good functional result in the long term.

The functional result was evaluated using the IKDC score, which was calculated 6 months, 12 months, and 18 months after ligamentoplasty. At the last follow-up, clinical evaluation was based on the joint amplitude of the knee, muscle triplicity (measured as the thigh circumference $10 \mathrm{~cm}$ above the patella) and the waiting time before resuming sports and professional activities.

\section{Statistical analysis}

The data were gathered using Excel software (Microsoft, Redmond, WA). Statistical analysis of the quantitative variables was comprised of calculating the means and the standard deviations. The quantitative variables were described by their frequency and corresponding percentage. The results from the two groups were compared using a Fisher's exact test and a Wilcoxon test for the quantitative variables. The significance threshold was set at 0.05 .

\section{Results}

\section{Demographic data}

During the relevant period, 100 patients underwent ACL ligamentoplasty, and 69 were included in this study: 36 in the ST4 group and 33 in the STG group. The average waiting time before surgery was one year (range: 1 month - 4 years). In the STG group, there were 10 competitive sports players who all played pivoting sports, 21 recreational sports players including 15 who played pivoting sports, and 2 active-duty soldiers In the ST4 group, there were 11 competitive sports players including 10 who played pivoting sports, 22 recreational sports players including 16 who played pivoting sports, and 3 active-duty soldiers. These data are entirely comparable between the two groups. The sex ratio favoured men in both groups: 3 in the ST4 group and 1.54 in the STG group. There were more women in the STG group, but the difference was not statistically significant. The average BMI was 25.1 in the ST4 group and 24.8 in the STG group ( $\mathrm{p}=0.8$ ). There were no significant differences in the age and military status of the patients between the two groups. Nine patients had meniscus tears in the ST4 group and 12 in the STG group. All the meniscus tears were sutured during the ligamentoplasty. The transplant diameter was significantly larger in the ST4 group (Table 1).

Table 1: Demographic, operative, and clinical data.

\begin{tabular}{|c|c|c|c|c|c|c|}
\hline Group & $\mathbf{n}$ & $\begin{array}{c}\text { Mean Age } \\
\text { (Range) }\end{array}$ & Military & $\begin{array}{c}\text { Diameter of the Trans- } \\
\text { plant }\end{array}$ & Subjective IKDC 18 months & $\begin{array}{c}\text { Objective IKDC 18 } \\
\text { months }\end{array}$ \\
\hline ST4 & 36 & $27.5(17-41)$ & 26 & 9.39 & $75 \% \mathrm{~A} / 17 \% \mathrm{~B}$ & 87.2 \\
\hline STG & 33 & $31(21-51)$ & 20 & 8 & $69 \% \mathrm{~A} / 21 \% \mathrm{~B}$ & 85.5 \\
\hline $\mathrm{p}$ & & 0.4 & 0.5 & $<0.001$ & 0.6 & 0.01 \\
\hline
\end{tabular}




\section{Isokinetic data}

At the $6^{\text {th }}$ month post-op, the strength deficit of the hamstring and quadriceps was not significantly different between the two groups, with an identical H/Q ratio (Table 2). The best functional results (IKDC>90) had muscular deficits close to $10 \%$ on average. This was found similarly in both groups. Thus, there is a correlation between the IKDC scores and the isokinetic test results. In contrast, the age, sex, operating technique, and surgeon had no impact on the clinical and isokinetic results.

Table 2: Isokinetic data 6 months after surgery.

\begin{tabular}{|c|c|c|c|}
\hline Group & H Defect & Q Defect & H/Q ratio \\
\hline ST4 & $19 \%$ & $9 \%$ & 0.8 \\
\hline STG & $21 \%$ & $11 \%$ & 0.8 \\
\hline P & 0.3 & 0.2 & 0.2 \\
\hline
\end{tabular}

\section{Clinical results}

At the last follow-up at 18 months, there were no amplitude limitations of the knee joint, except in the patient from the ST4 group who had an infection (the patient flexes to $115^{\circ}$ ) and no cases of quadriceps amyotrophy were noted in either group. There were no differences in IKDC scores between the two groups, but the subjective score was significantly better in the ST4 group (Table 1). The IKDC scores at 12 and 18 months were stable. The average waiting time before resuming sports was 5.9 months [4-13 months] for straight-line sports and 12.5 months [10 months-no resumption] for pivoting sports, with no significant differences between the groups. In the ST4 group, only one patient was not able to resume competition (football) and the patient who suffered an infection only resumed cycling. At 18 months, 10 patients had returned to competition including 9 pivoting sports, 23 recreational sports players had resumed practice including 16 pivoting sports, and 3 patients had returned to active duty. In the STG group, two patients did not resume competition (football in both cases) and one recreational player was not able to return to his former lever. At the last follow-up, there were 8 competitive sports players who all played pivoting sports, 23 recreational sports players including 16 who played pivoting sports, and 2 active-duty soldiers. These data are significantly identical between the two groups.

\section{Complications observed}

Five patients from the ST4 group had complications that required surgical revision: a post-operative haematoma, an early infection, and three cases of cyclops syndrome, Four complications arose in the STG group: two transplant ruptures and two cases of cyclops syndrome that required surgical revision. The infection and the iterative ruptures were associated with poor IKDC scores. One soldier affected by an iterative rupture did not regain his operational capacities.

\section{Discussion}

This work analyses the results of an ACL ligamentoplasty technique using a hamstring graft composed of only the semitendino- sus in a homogenous population in terms of age, sports level, and waiting time before surgery. The particularity of this series is that it included a majority of military patients (46 out of 69) who had specific requirements for sports and professional performance. The results were compared to a conventional ligamentoplasty technique using the semitendinosus and the gracilis. The hypothesis was that preserving the gracilis would limit the loss of hamstring strength and so facilitate functional recovery.

Firstly, it was observed that the diameter of the ST4 transplant was larger than that of a classic STG transplant. This is linked to the shortness of the latter transplant. The transplant goes $0.8 \mathrm{~mm}$ into the blind femoral tunnel and $1.3 \mathrm{~mm}$ into the tibial tunnel. Primary fixation is achieved using strips and titanium screws. This causes the diameter to be larger than it would be for a transplant made only from the semitendinosus. According to Marchand et al. [10], the diameter of an ST4 should be between 8 and $10 \mathrm{~mm}$. He reiterates the data of Magnussen, Mariscalco et al. [11] concerning the link between the diameter of the transplant and knee stability obtained from laximetric analyses (KT 1000). The diameter must be at least $8 \mathrm{~mm}$ in order to obtain effective stability over time. Our two groups met this criteria.

With regards to the functional results, the IKDC scores obtained from the two groups were comparable and agreed with the data from the literature: for instance, the meta-analysis of Lewis et al. [12] found 35\% in class A and 44\% in class B; Biau et al. [13] showed $31 \%$ in class A and $43 \%$ in class B and the study by Colombet et al. [14] found $50 \%$ in class A and $44 \%$ in class B. The ST4TLS technique therefore makes it possible to obtain good clinical results with an average subjective IKDC of 87 at 18 months. Similar results had already been obtained by Polignac et al. [15], who found an average IKDC score of 92 in a series of 82 patients treated with this technique. Excluding the patient who had a transplant rupture, the resumption of pivoting sports occurred at roughly one year, with a return to the former level of ability in 44 cases out of 46 . This is comparable to the results obtained by Plaweski et al. [16], who observed in their prospective study that $86.6 \%$ of patients regained their former level of sports practice. These good results may be connected to the patients' very high level of sports practice, particularly the military patients, who must regain their former sports level rapidly in order to return to their jobs and who received a specific effort training course.

In this series, the average muscle strength deficit was $20 \%$ for the hamstrings and $10 \%$ for the quadriceps, in both groups, with no significant differences. These results slightly contrast the data in the litterature. The study by Condouret et al. [2] noted an average deficit of $10 \%$ and the meta-analysis by Dauty et al. [17] showed a deficit of between 5-17\%. The results of Dujardin et al. [18] were closer to ours, with an average deficit of $17 \%$, but Parisaux et al. [19] observed a more marked deficit of $23 \%$ Yasuda et al. [20] even observed a decrease in hamstring strength until one year after surgery. Papandrea et al. [21] showed in an ultrasound study that the harvested hamstrings healed with high reinsertion, which explains the moderate decrease in strength after harvesting. In this study, 
no significant difference was shown between harvesting only the semitendinosus and the conventional practice of harvesting the semitendinosus and gracilis muscles. This contradicts the study by Kyung et al. [22], who found a smaller strength deficit with laminoplasties that only harvested the semitendinosus. In this case, obtaining a satisfying $\mathrm{H} / \mathrm{Q}$ ratio was evidence of good muscle recovery in open kinetic chain exercises [9]. It is clearly established that a dynamic equilibrium between antagonist muscles is an important element for better, long-term functional results [23,24].

In contrast to Krych et al. [25], we did not find any factors that were predictive of good function and isokinetic results, such as age, the existence of meniscus tears or chondral injuries, or the waiting time before surgery. These parameters were homogenous and had no influence on the clinical or isokinetic results. However, like Condouret et al. [2], we showed that the patients with the best IKDC scores were those who had a muscle strength deficit of less than $10 \%$. This confirms that optimal muscle recovery is an essential driver of a favourable outcome. It would be desirable to perform isokinetic testing at 3 months in order to detect marked muscular deficits early on and offer a tailored rehabilitation protocol [2]. It might also be useful to repeat this testing at one year in order to assess the efficacy of the posture and movement training. This would limit prolonged deficits and thus improve the functional results through optimal muscle recovery. In this series, the isokinetic results at 6 months were used by the rehabilitation physicians to create ability level groups during the effort training course that was offered between the $7^{\text {th }}$ and $8^{\text {th }}$ months post-op.

The main limitation of this study is obviously the retrospective nature of the functional assessment. Moreover, the inclusion of a majority of military patients who had a very high level of sports practice and professional requirements may have lead to a selection bias insomuch as these patients needed to achieve maximum recovery in order to return to their former positions. It is possible that different results would have been obtained with a population of civilian patients practicing recreational sports. In closing, it would have been interesting to compare these two groups with a third group of patients treated with a patellar tendon ligamentoplasty.

\section{Conclusion}

ACL ligamentoplasty using an ST4 graft offers satisfying functional results that are equivalent or even slightly superior to those obtained with conventional STG ligamentoplasty. It ensures that sports activities can be resumed in optimal conditions, even in military patients who are true professional athletes. This technique can, therefore, be proposed to operational staff.

\section{Conflict of Interest}

The authors have a declared interest with the company FH Orthopedics (C).

also be used in failed hip arthroplasty with intra-pelvic socket migration. This approach has some advantages: it allows the removal of the migrated cup with very good control of intrapelvic structures and it is less invasive than the transperitoneal approach.

\section{References}

1. Ageberg E, Roos HP, Silbernagel KG (2009) Knee extension and flexion muscle power after anterior cruciate ligament reconstruction with patellar tendon graft or hamstring tendons graft: a cross-sectionnal comparaison 3 years post-surgery. Knee Surg Sports Traumatol Arthrosc 17: 162-169.

2. Condouret J, Cohn J, Ferret JM (2008) Isokinetic assessment with two years follow-up of anterior cruciate ligament reconstruction with patellar tendon or hamstring tendons. Rev Chir Orthop Rep Appar Mot 94(8): 375-382.

3. Kyung HS, Lee HJ, Oh CW, Hong HP (2015) Comparaison of results after anterior cruciate ligament reconstruction using a four-strand single semitendinosus or a semitendinosus and gracilis tendon. Knee Surg Sports Traumatol Arthrosc 23(11): 3238-3243.

4. Parisaux JM, Boileau P, Desnuelle C (2004) Isokinetic evaluation of the knee flexor muscles after anterior cruciate ligament reconstruction using gracilis and semitendinosus tendon grafts. Rev Chir Ortho 90: 33-39.

5. Lipscomb AB, Johnston RK, Snyder RB, Warburton MJ, Gilbert PP (1982) Evaluation of hamstring strength following use of semitendinosus and gracilis tendons to reconstruct the anterior cruciate ligament. Am J Sports Med 10: 340-342.

6. Besnard N, Cazenave M, Hryszkiewicz J, Lagadec T (2010) Le TLS une technique novatrice de ligamentoplastie du genou au service d'une rééducation ambitieuse KS 509: 13-20.

7. Rochcongar P (2004) Isokinetic thigh muscle strengh in sports a review. Ann Readapt Med Phys 47: 274-281.

8. Gremeaux V, Forthomme B, Croisier JL (2009) Renforcement musculaire excentrique après plastie du LCA In : Croisier JL, Codine P (Eds.), Exercice musculaire excentrique Paris Masson, pp.103-112.

9. Middleton P, Petit H, Moreau V (2006) Equilibre musculaire : intérêt du ratio quadriceps concentrique : ischio-jambier excentrique. J Traum Sport 23: 43.

10. Marchand JB, Ruiz N, Coupry A, Bowen M (2016) Do graft diameter or patient age influence the results of ACL reconstruction. Knee Surg Sports Traumatol Arthrosc 24(9): 2998-3004.

11. Mariscalco MW, Flanigan DC, Mitchell J, Pedroza AD, Jones MH, et al. (2013) The influence of hamstring autograft size on patient-reported outcomes and risk of revision after anterior cruciate ligament reconstruction: a multicenter orthopaedic outcomes network (MOON) cohort study. Arthroscopy 29(12): 1948-1953.

12. Lewis PB, Parameswaran D, Rue JP, Bach BR (2008) Systematic review of single bundle ACL reconstruction outcomes. Am J Sports Med 10: 20282036.

13. Biau DJ, Tournoux C, Katsahian S, Schranz PJ, Nizard R (2006) Bone-patellar tendon-bone autografts versus hamstring autografts for reconstruction of anterior cruciate ligament: meta-analysis. BMJ 332: 9951001.

14. Colombet Ph, Allard M, Bousquet V, de Lavigne C, Flurin PH, et.al (2002) Anterior cruciate ligament reconstruction using four-strand semitendinosus and gracilis tendon grafts and metal interference screx fixation. Arthroscopy 18(3): 232-237.

15. Robert H, Limozin R, de Polignac T (2011) Single-bundle reconstruction in quadruple semi tendinosus graft of the ACL according to the TLS technique. Clinical results of a series of 74 knees with minimum 18 months follow-up. Orthop Traumatol Surg Res 97S: S40-S45.

16. Plaweski S, Rossi J, Merloz P (2009) Anterior cruciate ligament reconstruction: assessment of the hamstring autograft femoral, fixation using the endobutton CL. Orthop Traumatol Surg Res 95: 734-742.

17. Dauty M, Tortelier L, Rochcongar P (2004) Isokinetic and anterior cruciate ligament reconstruction with hamstrings or patellar tendon: analysis of literature. Int J Sports Med 25: 1-8. 
18. Dujardin D, Fontanin N, Geffrier A, Morel N, Mensa C, et.al (2015) Muscle recovery after ACL reconstruction with 4-strand semitendinosus graft harvested through either a posterior or anterior incision: A preliminary study Orthop Traumatol Surg Res 101: 346-349.

19. Parisaux JM, Boileau P, Desnuelle C Evaluation (2004) Isocinétique des muscles fléchisseurs du genou après greffe du ligament croisé antérieur utilisant le droit interne et le demi-tendineux. Rev Chir Orthop 90: 3339.

20. Yasuda K, Tsujino J, Ohkhoshi Y, Tanabe Y, Kaneda K (1995) Grafts morbidity with autogenous semi tendinosus and gracilis tendons. Am J Sports Med 23: 706-714.

21. Papandrea P, vulpani MC, Feretti A, Conteduca F (2000) Regeneration of the semitendinosus tendon harvest for anterior cruciate ligament reconstruction. Am J Sports Med 28: 556-561.
22. Kyung Hs, Lee HJ, Oh CW, Hong HP (2015) Comparaison of results after anterior cruciate ligament reconstruction using a four-strand single semitendinosus or a semitendinosus and gracilis tendon. Knee Sur Sports traumatol Arthrosc 23(11): 3238-3243.

23. Tashiro T, Kurosawa H, Kawakami A, Hikita A, Fukuri N (2003) Influence of medial hamstring tendon harvest on knee flexor strength after anterior cruciate ligament reconstruction. A detailed evaluation with comparison of single and double tendon. harvest Am J Sports Med 31: 522-529.

24. Haillotte G, Hardy A, Granger B, Noailles T, Khiami F (2017) Early strength recovery after anterior cruciate ligament reconstruction using the fascia lata. Orthop Traumatol Surg Res 03: 730-734.

25. Krych AJ, Woodcock JA, Morgan JA (2015) Factors associated with excellent6-month functional and isokinetic test results following ACL reconstruction. Knee Surg Sports Traumatol Arthrosc 23: 1053-1059.
Creative Commons Attribution 4.0 International License

For possible submissions Click Here

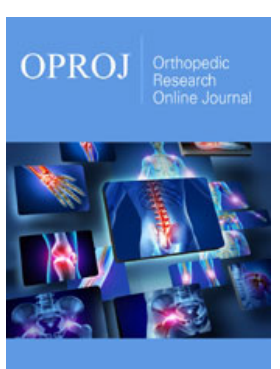

Orthopedic Research Online Journal

\section{Benefits of Publishing with us}

- High-level peer review and editorial services

- Freely accessible online immediately upon publication

- Authors retain the copyright to their work

- Licensing it under a Creative Commons license

- Visibility through different online platforms 